\title{
Direct measurements of growing amorphous order and non-monotonic dynamic correlations in a colloidal glass-former
}

\author{
K. Hima Nagamanasa ${ }^{1 \star}$, Shreyas Gokhale ${ }^{2}$, A. K. Sood ${ }^{2,3}$ and Rajesh Ganapathy ${ }^{3 \star}$
}

The transformation of flowing liquids into rigid glasses is thought to involve increasingly cooperative relaxation dynamics as the temperature approaches that of the glass transition. However, the precise nature of this motion is unclear, and a complete understanding of vitrification thus remains elusive. Of the numerous theoretical perspectives ${ }^{1-4}$ devised to explain the process, random first-order theory (RFOT; refs 2,5) is a well-developed thermodynamic approach, which predicts a change in the shape of relaxing regions as the temperature is lowered. However, the existence of an underlying 'ideal' glass transition predicted by RFOT remains debatable, largely because the key microscopic predictions concerning the growth of amorphous order and the nature of dynamic correlations lack experimental verification. Here, using holographic optical tweezers, we freeze a wall of particles in a two-dimensional colloidal glass-forming liquid and provide direct evidence for growing amorphous order in the form of a static point-to-set length. We uncover the non-monotonic dependence of dynamic correlations on area fraction and show that this non-monotonicity follows directly from the change in morphology and internal structure of cooperatively rearranging regions ${ }^{6,7}$. Our findings support RFOT and thereby constitute a crucial step in distinguishing between competing theories of glass formation.

In a seminal paper dated nearly fifty years ago, Adam and Gibbs ${ }^{8}$ associated the rapid growth of a supercooled liquid's relaxation time with a decrease in its configurational entropy $S_{c}$. Within RFOT, $S_{c}$ is related to the number of metastable minima in the free energy landscape of the liquid that can be explored by the system at a given temperature. This theory further claims that the supercooled liquid freezes into a mosaic whose domains correspond to configurations in these metastable minima ${ }^{1}$. The typical domain size is expected to diverge at the 'ideal' glass transition temperature, where $S_{\mathrm{c}}$ vanishes. The existence of a growing static 'mosaic' length scale that serves as a clear indicator of the glass transition is therefore intrinsic to RFOT (ref. 2), although a systematic procedure for measuring it from point-to-set correlations was established much later ${ }^{9}$. Since the findings of ref. 9, a variety of growing static length scales have been identified and computed in numerical simulations ${ }^{10-13}$. Of these, the point-to-set correlation length $\xi_{\text {PTS }}$ (ref. 10) is of central importance, as it follows directly from the mosaic picture. $\xi_{\text {PTS }}$ is measured by freezing a subset of particles in the liquid's equilibrium configuration, and examining their influence on the configuration of the remaining free particles. As such, when evaluated for appropriate pinning geometries, $\xi_{\text {PTS }}$ can provide an estimate of the typical domain size of the mosaic ${ }^{10}$. In addition, it has been shown analytically that a divergence in the relaxation time is indeed associated with a diverging $\xi_{\text {PTS }}$ (ref. 14). $\xi_{\text {PTS }}$ was first extracted in simulations by pinning all particles outside a spherical cavity and examining the configurations of free particles inside the cavity ${ }^{10}$. Subsequently, the growth of $\xi_{\text {PTS }}$ has been studied for various pinning geometries ${ }^{15}$ as well as in various simulated glass-formers ${ }^{16}$. Of particular importance is the case in which the pinned particles form a single amorphous wall. Using this geometry, in addition to $\xi_{\text {PTS }}$, recent simulations ${ }^{17}$ have computed a dynamic correlation length $\xi_{\text {dyn }}$ that evolves non-monotonically with temperature across the mode coupling crossover. It was surmised that this non-monotonicity reflects a change in the morphology of cooperatively rearranging regions (CRRs), which are string-like at high temperatures and compact close to the glass transition ${ }^{6}$. However, the crucial and long-standing microscopic predictions of RFOT pertaining to growing point-toset correlations and the morphology of CRRs remain untested in experiments. Point-to-set correlations cannot be investigated in atomic and molecular glass-formers, because the dynamics of their constituent particles cannot be traced, and it is not possible to freeze a subset of particles in an equilibrium configuration. These problems can be alleviated in colloidal glass-formers; in fact, the random pinning geometry was realized in a very recent experiment ${ }^{18}$. Given this advance in colloid experiments, testing the key predictions of RFOT directly in colloidal glass-formers would constitute a major step in unravelling the nature of the glass transition.

We performed optical video microscopy experiments on a binary mixture of small and large polystyrene colloids of diameters $\sigma_{\mathrm{S}}$ and $\sigma_{\mathrm{L}}$, respectively (see Methods for experimental details). As mentioned before, measuring point-to-set correlations requires pinning particles in an equilibrium configuration of the liquid, which is experimentally challenging. In colloidal systems, this can be realized by manipulating light fields using holographic optical tweezers $^{18,19}$. Here, we demonstrate the power of this technique by pinning an amorphous wall of particles in a two-dimensional colloidal glass-forming liquid. We first captured a bright-field image of the sample and extracted particle coordinates within a strip of width $\sim 2 \sigma_{\mathrm{L}}$ along the longer dimension of the field of view. We then calculated the hologram and fed it to a spatial light modulator (SLM), which in turn created traps at the desired positions. Further, the use of a SLM ensured that all the particles constituting the wall were frozen simultaneously (see Supplementary Movie 1). To ensure that the particles thus pinned are indeed a part of the liquid's equilibrium configuration, we superimposed the coordinates of these particles on time-averaged images of the sample in the

\footnotetext{
${ }^{1}$ Chemistry and Physics of Materials Unit, Jawaharlal Nehru Centre for Advanced Scientific Research, Jakkur, Bangalore 560064, India. ${ }^{2}$ Department of Physics, Indian Institute of Science, Bangalore 560012, India. ${ }^{3}$ International Centre for Materials Science, Jawaharlal Nehru Centre for Advanced Scientific Research, Jakkur, Bangalore 560064, India. *e-mail: manasakandula.jnu@gmail.com; rajeshg@jncasr.ac.in
} 


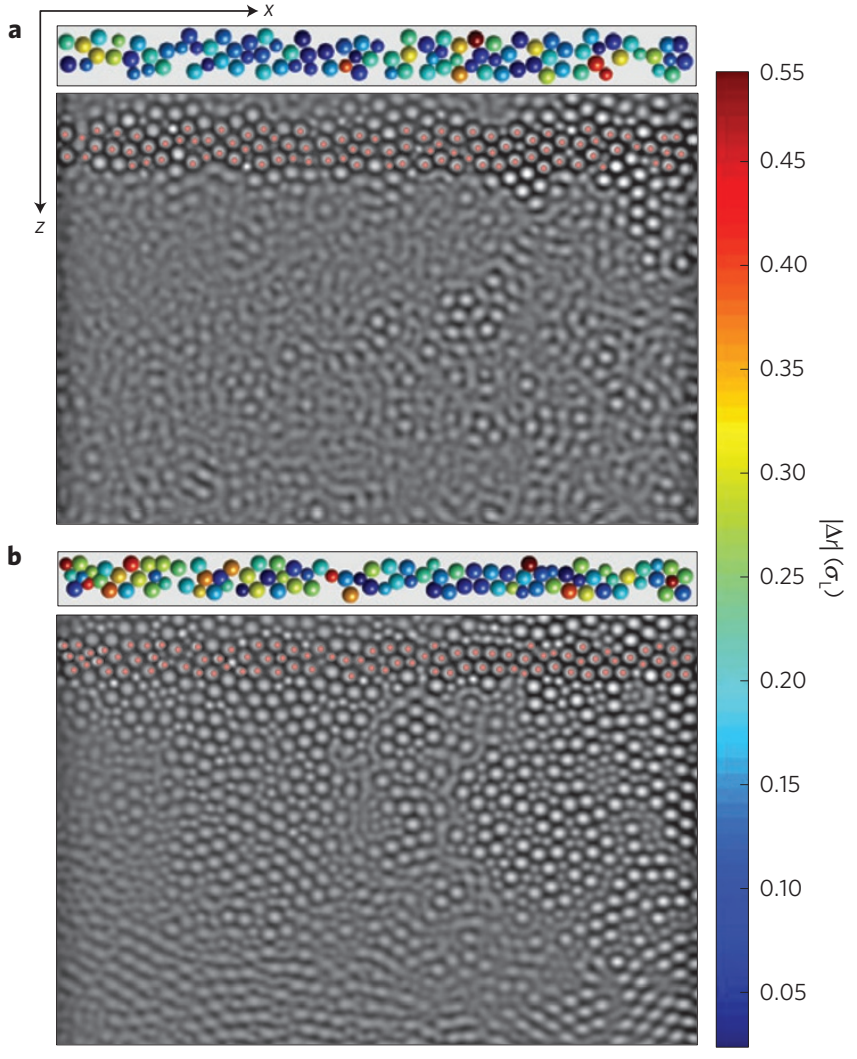

Figure 1 | Visualization of the amorphous wall. The underlying grey scale images have been generated by time-averaging snapshots over $30 \tau_{\alpha}$ for $\phi=0.68$ (a) and $\phi=0.76$ (b) for a pinned wall. $\tau_{\alpha}=12 \mathrm{~s}$ and $274 \mathrm{~s}$ for $\mathbf{a}$ and $\mathbf{b}$, respectively. The red circles correspond to the coordinates of the trapped particles that form the amorphous wall. The spheres at the top of the images in $\mathbf{a}$ and $\mathbf{b}$ constitute the pattern whose fast Fourier transform was fed into the spatial light modulator. Spheres are colour coded according to the distance between the input coordinates for creating traps and time-averaged particle positions in units of $\sigma_{\mathrm{L}}$

presence of the amorphous wall, for two different area fractions $\phi$ (Fig. 1). Particles forming the wall appear bright in the timeaveraged images owing to their negligible mobility, and can be easily identified. We observe that, by and large, the initial set of particle coordinates and the centres of pinned particles acquired from the time-averaged images are separated by a distance smaller than the cage size, which shows that particles forming the amorphous wall are pinned in an equilibrium configuration of the colloidal liquid. Interestingly, from the time-averaged images in Fig. 1, we see that clusters of immobile particles extend further away from the wall for $\phi=0.76$ than for $\phi=0.68$, suggesting that the influence of the wall is felt over longer distances with increasing area fraction.

We first extracted $\xi_{\text {PTS }}$ by calculating the total overlap function, $q_{c}(t, z)$, at various distances $z$ from the pinned wall ${ }^{17}$. Although this analysis was initially developed for three-dimensional systems, we have used its two-dimensional analogue for our colloidal glassformer. We divided the field of view into boxes of size $0.25 \sigma_{\mathrm{s}}$, and computed $q_{c}(t, z)$ for all boxes that lie at a fixed distance away from the wall, using the equation

$$
q_{\mathrm{c}}(t, z)=\frac{\sum_{i(z)}\left\langle n_{i}(t) n_{i}(0)\right\rangle}{\sum_{i(z)}\left\langle n_{i}(0)\right\rangle}
$$

where \langle\rangle correspond to time averaging, $i$ is the box index, $n_{i}(t)=1$ if the box contains a particle at time $t$ and $n_{i}(t)=0$ otherwise.
Figure 2a shows $q_{\mathrm{c}}(t, z)$ at various $z$ for $\phi=0.74$. The box size $0.25 \sigma_{\mathrm{S}}$ was chosen to be larger than the cage size, $\sim 0.14 \sigma_{\mathrm{S}}$, to avoid spurious overlap fluctuations due to cage rattling. Moreover, the chosen size is small enough to provide sufficient spatial resolution for the computation of $\xi_{\text {PTS }}$ and $\xi_{\text {dyn }}$. By definition, $q_{\mathrm{c}}(t, z)$ (equation (1)) measures the overlap between configurations at two different times at a given distance from the wall. As $q_{\mathrm{c}}(t, z)$ is insensitive to particle exchanges, in the limit of long times and large distances from the wall, it attains a finite asymptotic bulk value $q_{\text {rand }}=q_{\mathrm{c}}(t \rightarrow \infty, z \rightarrow \infty)$ corresponding to the probability of occupation of a box. Consistent with simulations ${ }^{17}$, we observe that the presence of the wall influences the asymptotic value of $q_{\mathrm{c}}(t \rightarrow \infty, z)=q_{\infty}(z)$, such that $q_{\infty}(z)>q_{\text {rand }}$ (Fig. 2a). In our experiments, $q_{\infty}(z)$ is estimated by averaging the saturation value of the overlap function over a 5-10 min time window ${ }^{20}$. We note that $q_{\mathrm{c}}(t, z)$ does not saturate for all $z$ within the experimental duration and hence, to extract $\xi_{\text {PTS }}$, we consider only those values of $z$ for which $q_{c}(t, z)$ attains saturation. As expected, we observe that $q_{\infty}(z)$ decreases with $z$ in the vicinity of the wall. This is also evident from Fig. 2a, where the $q_{\mathrm{c}}(t, z)$ profiles for large $z$ overlap almost completely. We observe that $q_{\infty}(z)-q_{\text {rand }}$ decays exponentially with $z$ (Fig. 2b and Supplementary Fig. 1), which allowed us to extract $\xi_{\text {PTS }}$ from the relation

$$
q_{\infty}(z)-q_{\mathrm{rand}}=B \exp \left(-z / \xi_{\mathrm{PTS}}\right)
$$

Having computed $\xi_{\text {PTS }}$, we computed $\xi_{\text {dyn }}$ from the self part of the overlap function, $q_{\mathrm{s}}(t, z)$ :

$$
q_{s}(t, z)=\frac{\sum_{i(z)}\left\langle n_{i}^{s}(t) n_{i}^{s}(0)\right\rangle}{\sum_{i(z)}\left\langle n_{i}^{s}(0)\right\rangle}
$$

where, once again, \langle\rangle correspond to time averaging, $i$ is the box index, and $n_{i}^{s}(t)=1$ if the box is occupied by the same particle at time $t$ and $n_{i}^{s}=0$ otherwise ${ }^{17} . q_{s}(t, z)$ is similar to the selfintermediate scattering function calculated for the wavevector corresponding to the box size. Unlike $q_{\mathrm{c}}(t, z), q_{\mathrm{s}}(t, z)$ (equation (3)) is sensitive to particle exchanges and reaches zero at long times, when all the particles undergo a displacement larger than the box size. Owing to its similarity with the self-intermediate scattering function, $q_{\mathrm{s}}(t, z)$ yields relaxation times $\tau_{\mathrm{s}}(z)$ at different distances $z$ from the wall ${ }^{17}$. Owing to the limited temporal resolution in our experiments, we defined $\tau_{\mathrm{s}}(z)$ as the time taken for $q_{\mathrm{s}}(t, z)$ to decay to 0.2 (ref. 21). Figure 2c shows $q_{\mathrm{s}}(t, z)$ at various $z$ for $\phi=0.74$. As expected, $\tau_{\mathrm{s}}(z)$ approaches its bulk value $\tau_{\mathrm{s}}^{\text {bulk }}$ for large $z$. In accordance with simulations ${ }^{17,22}$, we find that the dynamic length scale $\xi_{\text {dyn }}$ (Fig. 2d) can be extracted from the equation

$$
\ln \left(\tau_{\mathrm{s}}(z)\right)=\ln \left(\tau_{\mathrm{s}}^{\text {bulk }}\right)+B_{\mathrm{s}} \exp \left(-z / \xi_{\text {dyn }}\right)
$$

Having extracted $\xi_{\text {PTs }}$ and $\xi_{\text {dyn }}$ from overlap functions, we studied the variation of these length scales with the area fraction $\phi$ on approaching the glass transition (Fig. 2b,d). We find that, in concord with simulations ${ }^{17}, \xi_{\text {PTs }}$ grows monotonically with $\phi$ (Fig. 3a and Supplementary Fig. 1). This finding constitutes the first experimental evidence of growing point-to-set correlations in glass-forming liquids. We note that, as in simulations, the prefactor $B$ changes with $\phi$ (Fig. 2b). To ensure that the trend of growing amorphous order is not influenced by the variation in $B$, we defined a second static length scale $\xi_{\text {PTS-Int }}=B \xi_{\text {PTS }}$, which is also observed to increase with $\phi$ (ref. 17; Supplementary Fig. 1). Turning towards the dynamic length scale, $\xi_{\text {dyn }}$, we observe that it grows faster than $\xi_{\text {PTS }}$, as expected ${ }^{17}$. Most strikingly, however, $\xi_{\text {dyn }}$ exhibits a non-monotonic dependence on $\phi$ (Fig. 3a). This result is remarkable, as it is the first experimental observation of non-monotonicity in dynamic correlations. With the exception of the numerical results of Kob et al. ${ }^{17}$, all dynamic length scales 
a

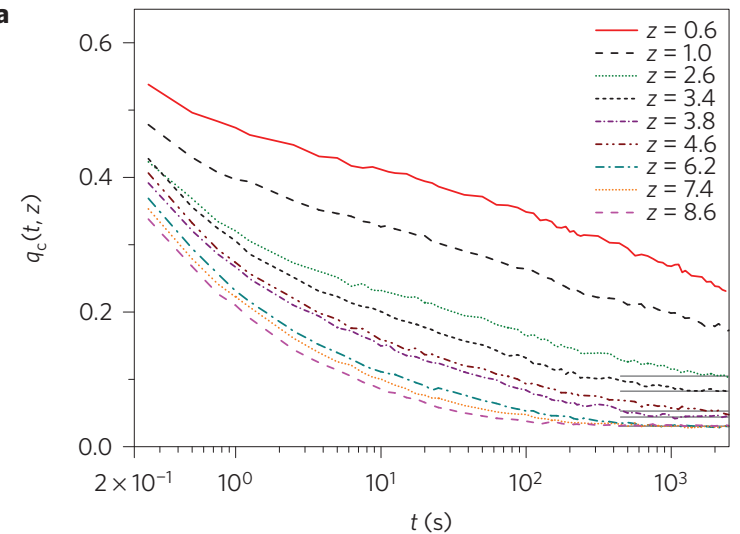

b

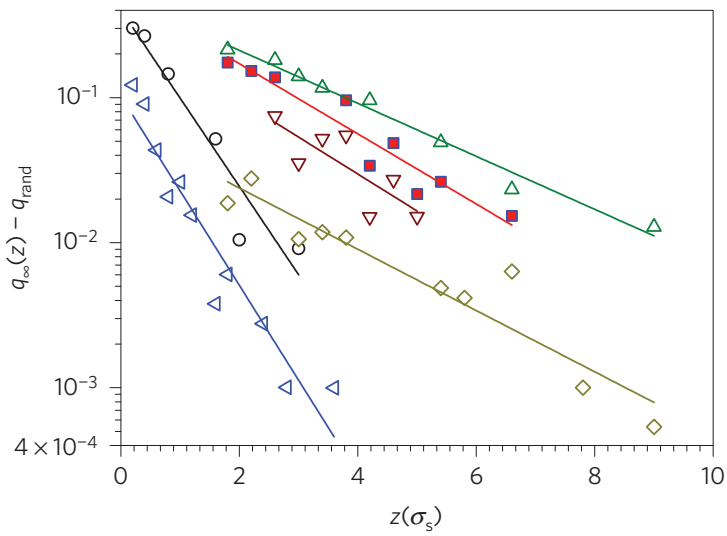

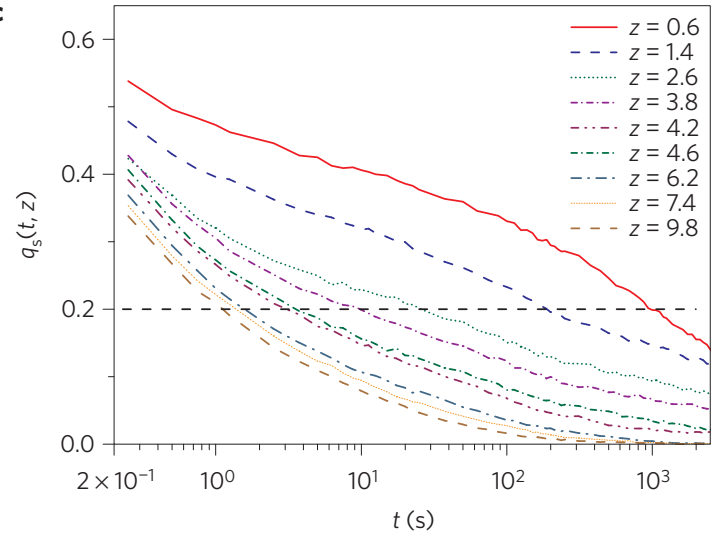

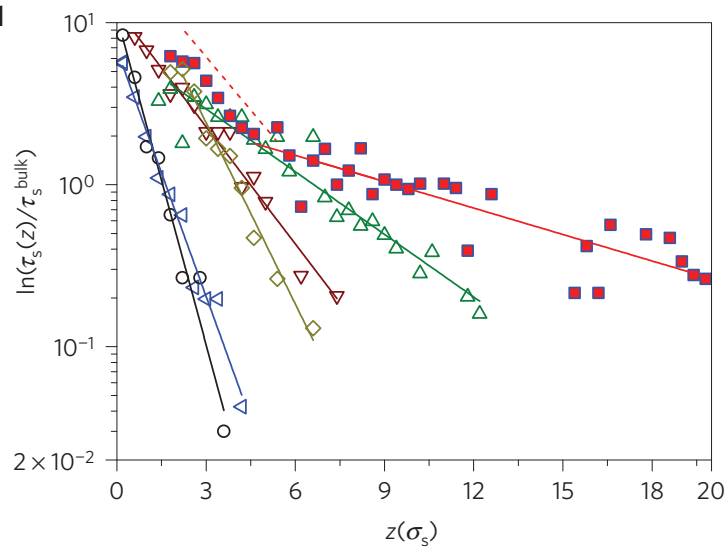

Figure 2 | Overlap functions and relaxation times. $\mathbf{a}$, The total overlap $q_{c}(t, z)$ for $\phi=0.74$. Different colours represent different $z$. The horizontal grey solid lines indicate $q_{\infty}(z)$, the value at which $q_{c}(t, z)$ saturates. $\mathbf{b}, q_{\infty}(z)-q_{\text {rand }}$ versus $z$ for $\phi=0.68$ (black open circles), $\phi=0.71$ (blue left triangles), $\phi=0.74$ (brown down triangles), $\phi=0.75$ (olive green diamonds), $\phi=0.76$ (red squares) and $\phi=0.79$ (green triangles). $\mathbf{c}$, The self overlap $q_{s}(t, z)$ for $\phi=0.74$. Different colours correspond to different $z$. The horizontal black dashed line corresponds to $q_{\mathrm{s}}(t, z)=0.2 \cdot \mathbf{d}, \ln \left(\tau_{\mathrm{s}}(z) / \tau_{\mathrm{s}}^{\text {bulk }}\right)$ as a function of $z$. The colours and symbols in $\mathbf{d}$ are identical to those in $\mathbf{b}$. In $\mathbf{b}$ and $\mathbf{d}$, the solid lines are exponential fits of the forms given in equations (2) and (4), respectively. In $\mathbf{d}$, for $\phi=0.76$ (red squares), $\xi_{\text {dyn }}$ was extracted from the asymptotic slope ${ }^{17}$. The red dashed line is a guide to the eye.

reported in the literature were seen to grow monotonically on approaching the glass transition ${ }^{23-26}$. The paucity in observations of the aforementioned non-monotonicity is due to the fact that not all dynamic length scales are expected to show non-monotonicity and the presence of a pinned wall seems to be crucial to this observation. Even in the presence of a pinned wall, it has been shown that the existence of non-monotonicity is dependent on the interaction potential ${ }^{27}$. An important point to note is that in the simulations of Kob and coworkers ${ }^{17}$, as well as our experiments, the maximum in $\xi_{\text {dyn }}$ occurs in the vicinity of the mode coupling crossover (Fig. 3a and Supplementary Fig. 2), strongly suggesting that our observations correspond to the same dynamic crossover seen in refs 27,28 . In ref. 17 , it has been speculated that the observed non-monotonicity is a consequence of a change in the morphology of CRRs across the mode coupling crossover, and is therefore consistent with RFOT. In particular, the authors of ref. 17 claim that the spatial inhomogeneity introduced by the wall makes $\xi_{\text {dyn }}$ sensitive not only to the number of particles in a CRR, but also to their arrangement into string-like or compact structures. A closer look at the relaxation profiles $\tau_{\mathrm{s}}(z)$ gives a preliminary indication in support of this claim. Whereas the profiles for $\phi<0.76$ as well as for $\phi=0.79$ are described well by a single exponential decay, $\tau_{\mathrm{s}}(z)$ exhibits two slopes for $\phi=0.76$ (Fig. 2d), which indicates the presence of multiple relaxation mechanisms associated with the morphology and internal structure of CRRs (ref. 17).

To test whether the non-monotonicity indeed stems from a change in the shapes of CRRs, we examined the nature of dynamical heterogeneities in our system. To ensure that the analysis is not influenced by the presence of a wall, we examined the shapes of CRRs in the corresponding unpinned system (see Methods for details). To define CRRs, we first identified the top $10 \%$ most mobile particles over various time intervals $\Delta t$ and clustered them based on nearest neighbour distances. As expected, the mean cluster size is maximal at a characteristic time $t^{*}$. On observing the shapes of these clusters defined over $\Delta t=t^{*}$, we find that, remarkably, the clusters are predominantly string-like at low $\phi$ and compact at high $\phi$ (Fig. 3b,d). To quantify this change in morphology, we computed the distribution $P(N N)$ of the number of mobile nearest neighbours of a mobile particle, following the protocol used in refs 23,29 (Fig. 3e). Interestingly, we observe that for $\phi<0.76, P(N N)$ exhibits a peak at $N N=2$, indicating string-like morphology $y^{30}$. For $\phi \geq 0.76$, the distribution becomes increasingly broader and the maximum shifts to $N N=3$, consistent with Fig. $3 \mathrm{~b}-\mathrm{d}$. To illustrate this point better, we have plotted the percentage of mobile particles that have greater than two mobile neighbours, given by $\int_{3}^{\infty} P(N N) \mathrm{d} N N$ (inset to Fig. 3e). We observe that the trend in this quantity indeed reflects the change in morphology of CRRs near $\phi_{\mathrm{MCT}}$ anticipated in the Fuzzy Sphere Model. We repeated the above analysis in the presence of a pinned wall, for regions located sufficiently far away from the wall, and observed a similar behaviour in $P(N N)$ (see Supplementary Fig. 3). These findings are consistent with RFOT, which predicts that string-like CRRs occur with greater frequency on decreasing $\phi$ and eventually become dominant below $\phi_{\mathrm{MCT}}$ (ref. 7). 
a

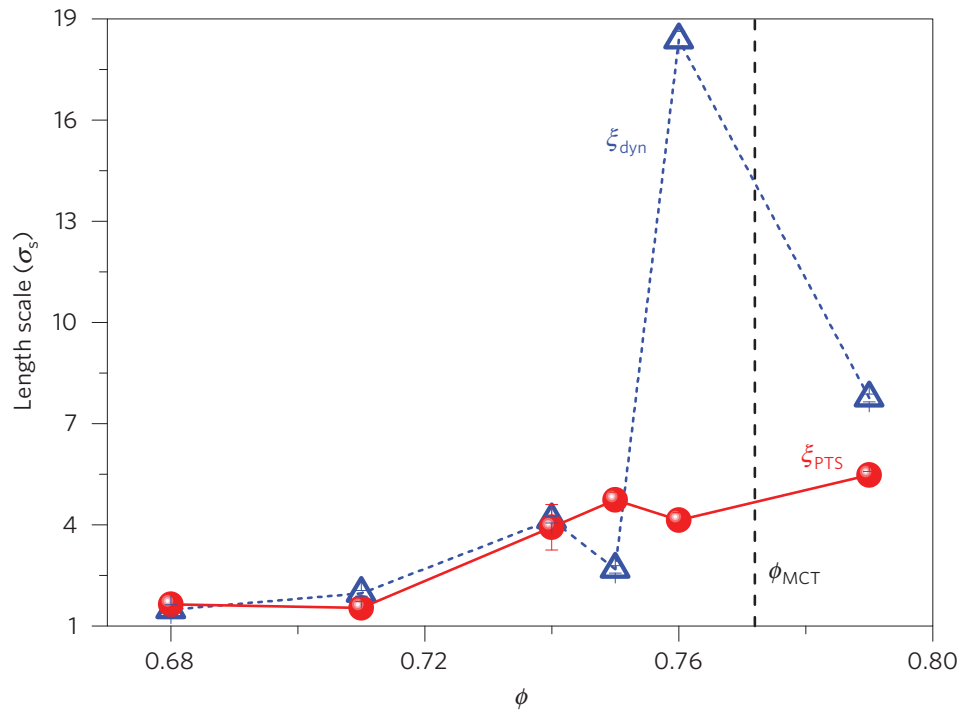

e

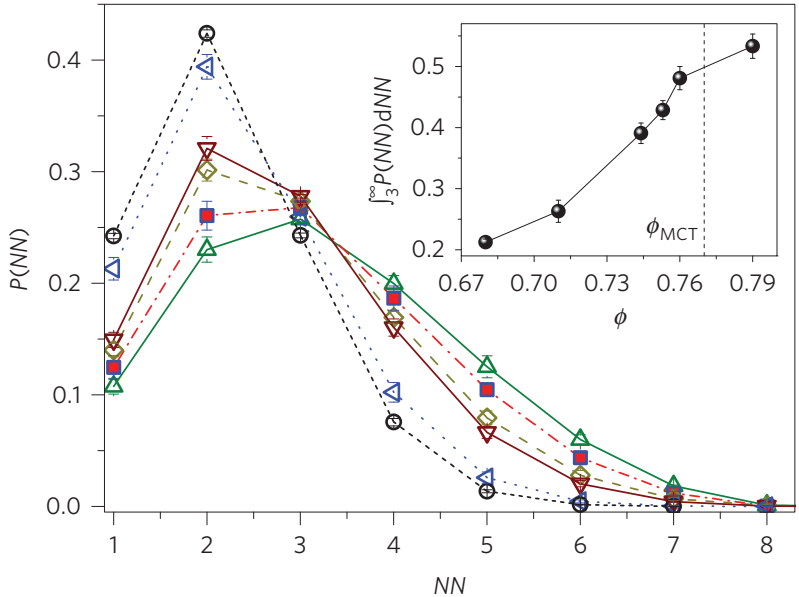

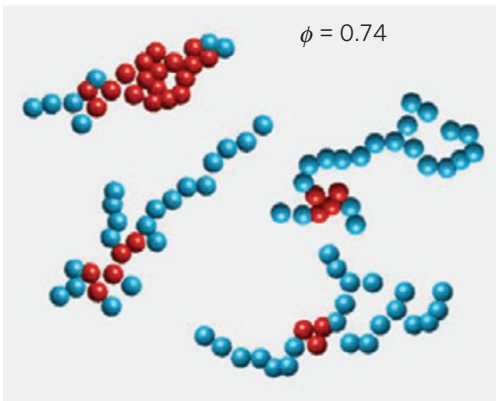

C

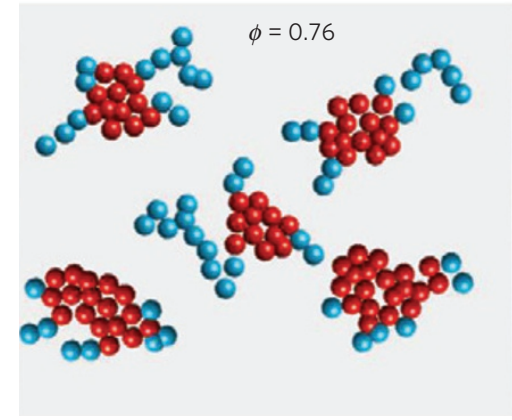

d

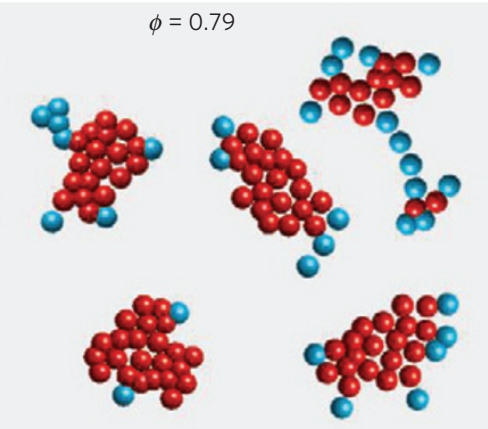

f

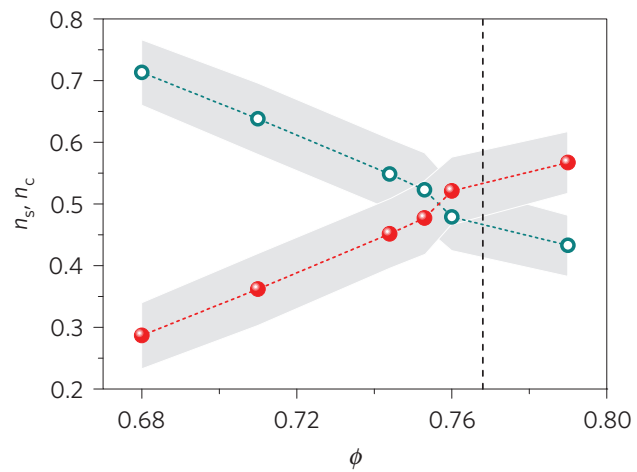

g

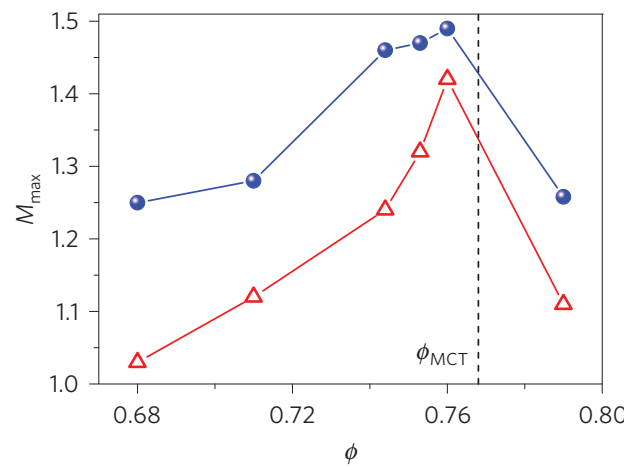

Figure 3 | Static and dynamic length scales and morphology of CRRs. a, Point-to-set length scale, $\xi_{\text {PTs, }}$ (red circles) and dynamic length scale, $\xi_{\text {dyn }}$ (blue triangles). The error bars have been obtained from the exponential fits. The dotted black line indicates the mode coupling crossover $\phi_{\mathrm{MCT}}$.

b-d, Representative cluster morphologies for 25 -particle clusters for $\phi=0.74, \phi=0.76$ and $\phi=0.79$ respectively. Core-like particles are shown in red and string-like particles are shown in light blue. e, Distribution of the number of mobile nearest neighbours, $P(N N)$, for $\phi=0.68$ (black open circles), $\phi=0.71$ (blue left triangles), $\phi=0.74$ (brown down triangles), $\phi=0.75$ (olive green diamonds), $\phi=0.76$ (red squares) and $\phi=0.79$ (green triangles). f, Fraction of string-like, $n_{s}$, (open green circles) and core-like, $n_{c}$, (red circles) particles as a function of $\phi$ for 25 -particle clusters. The grey shaded areas correspond to $50 \%$ confidence bands. $\mathbf{g}$, Maximum of the mobility transfer function $M(\Delta t), M_{\max }$, for small (blue circles) and large (red triangles) particles computed by considering the top $10 \%$ most mobile particles.

According to refs 6,7, the morphological changes in CRRs can be described by the 'Fuzzy Sphere Model. This model assumes CRRs to be composite objects that contain a compact core surrounded by a ramified string-like shell. Further, the string-like shell dominates at low $\phi$ and the compact core dominates at large $\phi$, with a smooth crossover between the activation barrier distributions for the two morphological types near $\phi_{\mathrm{MCT}}$. Interestingly, we observe that CRRs of a fixed size indeed contain a large fraction of string-like 
particles at low $\phi$ and a large fraction of core-like particles at high $\phi$ (Fig. 3b-d, also see Supplementary Movies 2-4). For further analysis, we quantitatively extracted the fractions $n_{\mathrm{s}}$ and $n_{\mathrm{c}}$ of stringlike and core-like particles respectively, within CRRs of various sizes (see Methods for a definition of string-like and core-like particles). Figure $3 \mathrm{f}$ shows the dependence of $n_{\mathrm{s}}$ and $n_{\mathrm{c}}$ on $\phi$ for CRRs containing $N=25$ particles (also see Supplementary Movies 2-4). We see that $n_{\mathrm{s}}$ and $n_{\mathrm{c}}$ indeed cross over near $\phi=0.76$, even for the bulk unpinned system (see Supplementary Fig. 4 for similar analysis for different values of $N$ ). Although we could sample only a limited number of $\phi$ values owing to experimental difficulties, it is evident from the data that the maximum in $\xi_{\text {dyn }}$ (Fig. 3a) coincides with the crossover in the morphology of CRRs (Fig. 3e,f). These findings therefore provide direct confirmation that the non-monotonicity in $\xi_{\text {dyn }}$ results from a change in the shape as well as internal structure of CRRs. Further, we observe that the average number of particles per cluster increases monotonically (Supplementary Fig. 5). This is consistent with previous studies on colloidal glass-formers ${ }^{23,24}$ and strongly suggests that length scales that grow monotonically on approaching the glass transition are sensitive only to the number of particles in a CRR and not to their arrangement within it. In a broader context, our results provide the first direct verification for the change in morphology and internal structure of CRRs across the mode coupling crossover ${ }^{6,7}$.

As a final point, we discuss our findings in the light of the dynamical facilitation (DF) approach ${ }^{3}$, a purely kinetic theory of glass transition that has recently garnered experimental support ${ }^{18,31}$. The DF theory states that structural relaxation takes place via the coordinated motion of localized mobile defects, whose concentration decreases on approaching the glass transition. Within the DF approach, string-like cooperative motion over $t^{*}$ arises hierarchically from the dynamics of these defects ${ }^{32}$. However, in its current form, the DF theory for atomistic glass-formers does not anticipate a crossover in the morphology of CRRs. A major difference between the RFOT and DF approaches is that the former emphasizes the importance of activated events that grow in size, whereas in the latter, relaxation is dominated by the facilitated dynamics of localized defects. The importance of facilitated dynamics can be quantified by evaluating the mobility transfer function $M(\Delta t)$ (ref. 33; see Supplementary Information for a definition of $M(\Delta t)$ and Supplementary Fig. 6 for the evolution of $M(\Delta t)$ with $\phi)$. The maximum $M_{\max }$ of $M(\Delta t)$ is expected to increase monotonically with $\phi$ if facilitation dominates structural relaxation. In the presence of nonfacilitated activated processes that become dominant close to the glass transition, $M_{\max }$ is expected to exhibit a maximum at the $\phi$ corresponding to the crossover from the facilitation-dominated regime to the activated-hopping regime ${ }^{34}$. Remarkably, we observe that, in our system, $M_{\max }$ shows a maximum at $\phi=0.76$ (Fig. $3 \mathrm{~g}$ and Supplementary Fig. 6), the very same $\phi$ at which $\xi_{\text {dyn }}$ shows a maximum (Fig. 3a) and the morphology of CRRs changes from string-like to compact (Fig. 3e,f). We note that a similar dependence of $M_{\max }$ on $\phi$ has been observed in a completely different colloidal system as well ${ }^{18}$. The observed $\phi$ dependence of $M_{\max }$ is qualitatively consistent with RFOT, where facilitation is a secondary relaxation process that diminishes in importance on approaching the glass transition ${ }^{35}$, but is in stark contrast with predictions of the DF theory.

Our observation of a growing point-to-set correlation length, $\xi_{\text {PTS }}$ (Fig. 3a), is consistent with the prediction of RFOT, although it does not rule out competing theoretical scenarios ${ }^{36,37}$. However, at present, the non-monotonic density dependence of $\xi_{\text {dyn }}$ (Fig. 3a) and the concomitant change in the morphology and internal structure of CRRs (Fig. 3e,f) can be rationalized only within the framework of RFOT. Crucially, the morphological crossover in CRRs as well as the maximum in the $\phi$ dependence of $M_{\max }$ find no natural explanation within the prominent competing framework of the DF theory. The non-monotonicity in $M_{\max }$ (Fig. 3g) observed here as well as in ref. 18 may be associated with the diminishing role of facilitated dynamics in governing structural relaxation, rather than finite size effects ${ }^{34}$. Indeed, reconciling these findings within the facilitation paradigm poses an exciting challenge for future theory, experiments and simulations. A promising course in this direction would be to examine the influence of a pinned wall on facilitated dynamics of localized defects. Given that we see signatures of increasing cluster size of immobile particles with $\phi$ in the time-averaged images shown in Fig. 1a, it would be fascinating to explore connections between the regions of slow dynamics and the static and dynamic length scales extracted here. It would also be instructive to investigate whether our results are consistent with other thermodynamic frameworks, such as geometric frustrationbased models ${ }^{4}$. We expect our findings to engender future research aimed at addressing these unresolved issues on the theoretical, numerical as well as experimental fronts.

\section{Methods}

Experimental details. Our system consisted of a binary mixture of $N_{\mathrm{S}}$ small and $N_{\mathrm{L}}$ large polystyrene particles of diameters $\sigma_{\mathrm{S}}=1.05 \mu \mathrm{m}$ and $\sigma_{\mathrm{L}}=1.4 \mu \mathrm{m}$, respectively. The particle size ratio $\sigma_{\mathrm{L}} / \sigma_{\mathrm{S}}=1.3$ and number ratio $N_{\mathrm{S}} / N_{\mathrm{L}}=1.23$ provided sufficient geometric frustration to prevent crystallization. The samples were loaded into a wedge-shaped cell ${ }^{18}$ and the desired area fractions $\phi$ were attained by sedimentation of the sample to the monolayer-thick region of the wedge. The typical waiting time before data collection was $\sim 8-10 \mathrm{~h}$, which is several times larger than $\tau_{\alpha}$ for all $\phi<0.79$. Samples were imaged using a Leica DMI $6000 \mathrm{~B}$ optical microscope with a $\times 100$ objective (Plan apochromat, NA 1.4, oil immersion) and images were captured at frame rates ranging from $3.3 \mathrm{fps}$ to $5 \mathrm{fps}$ for $1-1.5 \mathrm{~h}$, depending on the value of $\phi$. The size of the viewing region is $44 \sigma \times 33 \sigma$, with $\sigma=\left(\sigma_{\mathrm{S}}+\sigma_{\mathrm{L}}\right) / 2$. The holographic optical tweezers set-up consisted of a linearly polarized constant power $(800 \mathrm{~mW}) \mathrm{CW}$ laser (Spectra-Physics, $\lambda=1,064 \mathrm{~nm}$ ) and the optical traps were created using a SLM $(512 \times 512,100 \mathrm{fps}$ refresh rate, Boulder Nonlinear Systems). Standard Matlab algorithms ${ }^{38}$ were used to generate particle trajectories and subsequent analysis was performed using codes developed in-house. The particle tracking resolution is $0.08 \mu \mathrm{m}$ and the mean squared displacement curves for all $\phi$ lie above the tracking resolution (Supplementary Fig. 7). For all $\phi \leq 0.76$, the duration of the experiment corresponds to several times $\tau_{\alpha}$, where $\tau_{\alpha}$ is the time at which the self-intermediate scattering function $F_{s}(q, t)$ drops to $1 /$ e for $q=2 \pi / \sigma$. For $\phi=0.79$, the available experimental data are not sufficient to extract $\tau_{\alpha}$, although the waiting time is six times longer than the duration of the experiment. The presence of a pinned wall does not allow for sample drift corrections. To overcome this issue, we have ensured that the cell was left undisturbed for 8-10 h before recording each data set. The typical drift in our data over the entire duration of the experiment $(1-1.5 \mathrm{~h})$ is small $-\sim 0.5 \sigma_{\mathrm{L}}$ in the $x$-direction and $\sim 0.1 \sigma_{\mathrm{L}}$ in the $z$-direction. Further, for every $\phi$, we have also recorded data in the absence of pinning, immediately after capturing the data in the presence of a pinned wall. We find that the estimate for $\phi_{\mathrm{MCT}}$ for the data in the absence of pinning, after appropriately subtracting the drift, is almost identical to that for data captured far away from the wall in the presence of pinning (see Supplementary Fig. 2)

Reference for measuring $z$. To set the reference for measuring $z$, we first calculated overlap functions for all the boxes lying within strips of width $0.5 \sigma_{\mathrm{S}}$, parallel to the wall-that is, along the $x$-axis-for the entire image. From these overlap functions, we estimated the $z$-coordinate for which the overlaps exhibit no decay and labelled that as the centre of the wall. Given that the wall is approximately two particle diameters wide, the overlap does not decay with time only for a few strips of $0.5 \sigma_{\mathrm{S}}$ away from the centre of the wall. We have chosen the centre of the last strip for which the overlap function does not exhibit a decaying profile with time as the reference from which to measure $z$. The error in estimating the $z=0$ line is thus $0.5 \sigma_{\mathrm{S}}$.

Procedure for identifying string-like and core-like particles in a CRR. We labelled particles in a given cluster as string-like or core-like based on the number of nearest and next-nearest neighbours using the procedure described below. First, we identified all the particles that have more than two nearest neighbours, $i_{N N>2}$, where $i$ is the particle index. This set contains particles that form the core of the CRR as well as the particles that connect the compact core to the strings emerging from it. A particle ' $j$ ' is labelled core-like, only if it has at least two $i_{N N>2}$ neighbours. The remaining particles are considered to be string-like.

Supplementary Fig. 4 shows the fraction of core-like particles, $n_{\mathrm{c}}$, and string-like particles, $n_{\mathrm{s}}$, for different cluster sizes. Remarkably, we find that for all cluster sizes $n_{\mathrm{c}}$ increases, whereas $n_{\mathrm{s}}$ decreases with increasing $\phi$. For the range of cluster 
sizes studied here, we see that the crossover in $n_{\mathrm{c}}$ and $n_{\mathrm{s}}$ systematically shifts to lower $\phi$ with increasing $N$, although it continues to remain in the vicinity of $\phi=0.76$

Received 22 August 2014; accepted 20 February 2015; published online 13 April 2015

\section{References}

1. Berthier, L. \& Biroli, G. Theoretical perspective on the glass transition and amorphous materials. Rev. Mod. Phys. 83, 587-645 (2011).

2. Kirkpatrick, T. R., Thirumalai, D. \& Wolynes, P. G. Scaling concepts for the dynamics of viscous liquids near an ideal glassy state. Phys. Rev. A 40, 1045-1054 (1989).

3. Chandler, D. \& Garrahan, J. P. Dynamics on the way to forming glass: Bubbles in space-time. Annu. Rev. Phys. Chem. 61, 191-217 (2010).

4. Tarjus, G., Kivelson, S. A., Nussinov, Z. \& Viot, P. The frustration-based approach of supercooled liquids and the glass transition: A review and critical assessment. J. Phys. Condens. Matter. 17, R1143 (2005).

5. Lubchenko, V. \& Wolynes, P. G. Theory of structural glasses and supercooled liquids. Annu. Rev. Phys. Chem. 58, 235-266 (2007).

6. Stevenson, J. D., Schmalian, J. \& Wolynes, P. G. The shapes of cooperatively rearranging regions in glass-forming liquids. Nature Phys. 2, 268-274 (2006).

7. Stevenson, J. D. \& Wolynes, P. G. A universal origin for secondary relaxations in supercooled liquids and structural glasses. Nature Phys. 6, 62-68 (2010).

8. Adam, G. \& Gibbs, J. H. On the temperature dependence of cooperative relaxation properties in glass-forming liquids. J. Chem. Phys. 43, 139-146 (1965).

9. Bouchaud, J-P. \& Biroli, G. On the Adam-Gibbs-Kirkpatrick-ThirumalaiWolynes scenario for the viscosity increase in glasses. J. Chem. Phys. 121, 7347-7354 (2004).

10. Biroli, G., Bouchaud, J-P., Cavagna, A., Grigera, T. S. \& Verrocchio, P. Thermodynamic signature of growing amorphous order in glass-forming liquids. Nature Phys. 4, 771-775 (2008).

11. Tanaka, H., Kawasaki, T., Shintani, H. \& Watanabe, K. Critical-like behaviour of glass-forming liquids. Nature Mater. 9, 324-331 (2010).

12. Kurchan, J. \& Levine, D. Order in glassy systems. J. Phys. A 44, 035001 (2011).

13. Karmakar, S., Lerner, E. \& Procaccia, I. Direct estimate of the static length-scale accompanying the glass transition. Physica A 391, 1001-1008 (2012).

14. Montanari, A. \& Semerjian, G. Rigorous inequalities between length and time scales in glassy systems. J. Stat. Phys. 125, 23-54 (2006).

15. Berthier, L. \& Kob, W. Static point-to-set correlations in glass-forming liquids. Phys. Rev. E 85, 011102 (2012).

16. Hocky, G. M., Markland, T. E. \& Reichman, D. R. Growing point-to-set length scale correlates with growing relaxation times in model supercooled liquids. Phys. Rev. Lett. 108, 225506 (2012).

17. Kob, W., Roldán-Vargas, S. \& Berthier, L. Non-monotonic temperature evolution of dynamic correlations in glass-forming liquids. Nature Phys. 8, 164-167 (2012)

18. Gokhale, S., Nagamanasa, K. H., Ganapathy, R. \& Sood, A. K. Growing dynamical facilitation on approaching the random pinning colloidal glass transition. Nature Commun. 5, 4685 (2014).

19. Irvine, W. T. M., Hollingsworth, A. D., Grier, D. G. \& Chaikin, P. M. Dislocation reactions, grain boundaries and irreversibility in two dimensional lattices using topological tweezers. Proc. Natl Acad. Sci. USA 110, 15544-15548 (2013).

20. Charbonneau, P. \& Tarjus, G. Decorrelation of the static and dynamic length scales in hard-sphere glass formers. Phys. Rev. E 87, 042305 (2013).

21. Starr, F. W., Douglas, J. F. \& Sastry, S. The relationship of dynamical heterogeneity to the Adam-Gibbs and random first-order transition theories of glass formation. J. Chem. Phys. 138, 12A541 (2013).
22. Scheidler, P., Kob, W. \& Binder, K. Cooperative motion and growing length scales in supercooled confined liquids. Europhys. Lett. 59, 701-707 (2002).

23. Weeks, E. R., Crocker, J. C., Levitt, A. C., Schofield, A. \& Weitz, D. A. Three-dimensional direct imaging of structural relaxation near the colloidal glass transition. Science 287, 627-631 (2000).

24. Kegel, W. K. \& van Blaaderen, A. Direct observation of dynamical heterogeneities in colloidal hard-sphere suspensions. Science $\mathbf{2 8 7}$ 290-293 (2000).

25. Berthier, L. et al. Direct experimental evidence of a growing length scale accompanying the glass transition. Science 310, 1797-1800 (2005).

26. Flenner, E. \& Szamel, G. Characterizing dynamic length scales in glass-forming liquids. Nature Phys. 8, 696-697 (2012).

27. Hocky, G. M., Berthier, L., Kob, W. \& Reichman, D. R. Crossovers in the dynamics of supercooled liquids probed by an amorphous wall. Phys. Rev. E 89, 052311 (2014)

28. Flenner, E., Staley, H. \& Szamel, G. Universal features of dynamic heterogeneity in supercooled liquids. Phys. Rev. Lett. 112, 097801 (2014).

29. Zhang, Z., Yunker, P. J., Habdas, P. \& Yodh, A. G. Cooperative rearrangement regions and dynamical heterogeneities in colloidal glasses with attractive versus repulsive interactions. Phys. Rev. Lett. 107, 208303 (2011).

30. Donati, C. et al. Stringlike cooperative motion in a supercooled liquid. Phys. Rev. Lett. 80, 2338-2341 (1998).

31. Mishra, C. K., Nagamanasa, K. H., Ganapathy, R., Sood, A. K. \& Gokhale, S Dynamical facilitation governs glassy dynamics in suspensions of colloidal ellipsoids. Proc. Natl Acad. Sci. USA 111, 15362-15367 (2014).

32. Keys, A. S., Hedges, L. O., Garrahan, J. P., Glotzer, S. C. \& Chandler, D. Excitations are localized and relaxation is hierarchical in glass-forming liquids. Phys. Rev. X 1, 021013 (2011).

33. Vogel, E. \& Glotzer, S. C. Spatially heterogeneous dynamics and dynamic facilitation in a model of viscous silica. Phys. Rev. Lett. 92, 255901 (2004).

34. Elmatad, Y. S. \& Keys, A. S. Manifestations of dynamical facilitation in glassy materials. Phys. Rev. E 85, 061502 (2012).

35. Bhattacharyya, S. M., Bagchi, B. \& Wolynes, P. G. Facilitation, complexity growth, mode coupling, and activated dynamics in supercooled liquids. Proc. Natl Acad. Sci. USA 105, 16077-16082 (2008).

36. Jack, R. L. \& Garrahan, J. P. Caging and mosaic length scales in plaquette spin models of glasses. J. Chem. Phys. 123, 164508 (2005).

37. Jack, R. L. \& Berthier, L. Random pinning in glassy spin models with plaquette interactions. Phys. Rev. E 85, 021120 (2012).

38. Crocker, J. C. \& Grier, D. G. Methods of digital video microscopy for colloida studies. J. Colloid Interface Sci. 179, 298-310 (1996)

\section{Acknowledgements}

The authors thank W. Kob for illuminating discussions. K.H.N. thanks the Council for Scientific and Industrial Research (CSIR), India for a Senior Research Fellowship. S.G. thanks CSIR, India for a Shyama Prasad Mukherjee Fellowship. A.K.S. thank Department of Science and Technology (DST), India for support under a J. C. Bose Fellowship and R.G. thanks the International Centre for Materials Science (ICMS) and the Jawaharlal Nehru Centre for Advanced Scientific Research (JNCASR) for financial support.

\section{Author contributions}

K.H.N., S.G., A.K.S. and R.G. designed the research, analysed the data and wrote the paper. K.H.N. performed the experiments.

\section{Additional information}

Supplementary information is available in the online version of the paper. Reprints and permissions information is available online at www.nature.com/reprints.

Correspondence and requests for materials should be addressed to K.H.N. or R.G.

\section{Competing financial interests}

The authors declare no competing financial interests. 